\title{
Supercharacters of unipotent groups defined by involutions (extended abstract)
}

\author{
Scott Andrews \\ University of Colorado Boulder, USA
}

\begin{abstract}
We construct supercharacter theories of finite unipotent groups in the orthogonal, symplectic and unitary types. Our method utilizes group actions in a manner analogous to that of Diaconis and Isaacs in their construction of supercharacters of algebra groups. The resulting supercharacter theories agree with those of André and Neto in the case of the unipotent orthogonal and symplectic matrices and generalize to a large collection of subgroups. In the unitary group case, we describe the supercharacters and superclasses in terms of labeled set partitions and calculate the supercharacter table.
\end{abstract}

Résumé. Nous construisons les théories de super-caractères des groupes finis unipotents avec les types orthonormales, symplectiques et unitaires. Notre méthode utilise les actions des groupes d'une manière analogue à Diaconis et Isaccs dans leur construction des super-caractères des groupes algébriques. Les théories de super-caractères qui en résultent sont en accord avec ceux d'André et de Neto dans le cas des matrices unipotentes orthonormales et symplectiques. Elles généralisent en une grande sous-collection de sous-groupes. Dans le cas des groupes unitaires, nous décrivons les super-caractères et les superclasses dans les termes de partages d'ensembles étiquetés et nous calculons le tableau des super-caractères.

Keywords: supercharacter, representation theory, unipotent group, labeled set partition

\section{Introduction}

For $q$ a power of a prime, let $U T_{n}\left(\mathbb{F}_{q}\right)$ denote the group of unipotent $n \times n$ upper triangular matrices over the $q$ element field $\mathbb{F}_{q}$. Classifying the irreducible representations of $U T_{n}\left(\mathbb{F}_{q}\right)$ is known to be a "wild" problem (see [GKP ${ }^{+}$90]). In [And95, And02], André constructs a set of characters, referred to as "basic characters," such that each irreducible character of $U T_{n}\left(\mathbb{F}_{q}\right)$ occurs with nonzero multiplicity in exactly one basic character. These characters can be thought of as a coarser approximation of the irreducible characters of $U T_{n}\left(\mathbb{F}_{q}\right)$. Diaconis-Isaacs generalize the idea of a basic character to a "supercharacter" of an arbitrary finite group in [DI08]. They also construct supercharacter theories for all finite algebra groups $G$, which are subgroups of $U T_{n}\left(\mathbb{F}_{q}\right)$ such that $\{g-1 \mid g \in G\}$ is an $\mathbb{F}_{q}$-algebra. In the case that $G=$ $U T_{n}\left(\mathbb{F}_{q}\right)$, the constructions of Andre and of Diaconis-Isaacs produce the same supercharacter theory. The two constructions use different techniques; André constructs basic characters by inducing linear characters from certain subgroups of $U T_{n}\left(\mathbb{F}_{q}\right)$, whereas Diaconis-Isaacs utilize the two-sided action of $U T_{n}\left(\mathbb{F}_{q}\right)$ on the associative algebra of strictly upper triangular matrices.

1365-8050 @ 2014 Discrete Mathematics and Theoretical Computer Science (DMTCS), Nancy, France 
André-Neto have modified André's earlier construction to the unitriangular groups in types $B, C$ and $D$ in [AN06, AN09a, AN09b]. In this paper, we generalize these supercharacter theories in a manner analogous to the type $A$ construction of Diaconis-Isaacs. The construction in [AN06, AN09a, AN09b] uses the idea of a "basic subset of roots" to induce linear characters from certain subgroups of the full unitriangular group. Our construction instead utilizes actions of $U T_{n}\left(\mathbb{F}_{q}\right)$ on the Lie algebras of the unitriangular groups in types $B, C$ and $D$ to define superclasses and supercharacters. One advantage of our method is that it works in situations where the idea of a basic subset of roots does not make sense, such as the case of the unipotent radical of a parabolic subgroup.

Given a pattern subgroup $G$ (an algebra group such that $\{g-1 \mid g \in G\}$ has a basis of elementary matrices) of the unipotent upper triangular matrices and a subgroup $U$ of $G$ defined by an anti-involution of $G$, we construct a supercharacter theory. The anti-involution of $G$ induces an action of $G$ on the Lie algebra of $U$, which we use to construct the superclasses and supercharacters. The examples that naturally fall into this context include the unipotent orthogonal, symplectic and unitary groups. Let $J$ denote the $n \times n$ matrix with ones on the anti-diagonal and zeroes elsewhere, and let

$$
\Omega=\left(\begin{array}{cc}
0 & J \\
-J & 0
\end{array}\right)
$$

For $q$ a power of an odd prime, define

$$
\begin{aligned}
U O_{n}\left(\mathbb{F}_{q}\right) & =\left\{g \in U T_{n}\left(\mathbb{F}_{q}\right) \mid g^{-1}=J g^{t} J\right\} \quad \text { and } \\
U S p_{2 n}\left(\mathbb{F}_{q}\right) & =\left\{g \in U T_{2 n}\left(\mathbb{F}_{q}\right) \mid g^{-1}=-\Omega g^{t} \Omega\right\} .
\end{aligned}
$$

The groups $U O_{n}\left(\mathbb{F}_{q}\right)$ are the unipotent groups of types $B$ and $D$, and the groups $U S p_{2 n}\left(\mathbb{F}_{q}\right)$ are the unipotent groups of type $C$. Note that these groups are each defined by an anti-involution of $U T_{n}\left(\mathbb{F}_{q}\right)$; our construction produces the supercharacter theories constructed by André-Neto in [AN06, AN09a, AN09b].

We can also construct supercharacter theories of the unipotent unitary groups. For $g \in U T_{n}\left(\mathbb{F}_{q^{2}}\right)$, define $\bar{g}$ by $(\bar{g})_{i j}=\left(g_{i j}\right)^{q}$. Let

$$
U U_{n}\left(\mathbb{F}_{q^{2}}\right)=\left\{g \in U T_{n}\left(\mathbb{F}_{q^{2}}\right) \mid g^{-1}=J \bar{g}^{t} J\right\} .
$$

The group $U U_{n}\left(\mathbb{F}_{q^{2}}\right)$ is the group of unipotent unitary $n \times n$ matrices over $\mathbb{F}_{q^{2}}$. As $U U_{n}\left(\mathbb{F}_{q^{2}}\right)$ is a subgroup of $U T_{n}\left(\mathbb{F}_{q^{2}}\right)$ which is defined by an anti-involution, we get a supercharacter theory from the action of $U T_{n}\left(\mathbb{F}_{q^{2}}\right)$ on the Lie algebra of $U U_{n}\left(\mathbb{F}_{q^{2}}\right)$.

The supercharacters and superclasses of the unipotent unitary group are indexed by labeled set partitions. This has been shown for types $A, B, C$ and $D$ in [And02, AN09a]. In Section 5 , we describe this indexing, and calculate the supercharacter table of the unipotent unitary group in terms of labeled set partitions. The supercharacter table demonstrates Ennola duality, as the entries are obtained from the supercharacter table of $U T_{n}\left(\mathbb{F}_{q}\right)$ by formally replacing ' $q$ ' with ' $-q$ '. 


\section{Preliminaries}

\subsection{Supercharacter theories}

The idea of a supercharacter theory of an arbitrary finite group was introduced by Diaconis-Isaacs in [DI08]. Let $G$ be a finite group and let $\operatorname{Irr}(G)$ denote the set of irreducible characters of $G$. For a subset $X \subseteq \operatorname{Irr}(G)$, define

$$
\sigma_{X}=\sum_{\chi \in X} \chi(1) \chi
$$

Suppose that $\mathcal{K}$ is a partition of $G$ into unions of conjugacy classes and $\mathcal{X}$ is a partition of $\operatorname{Irr}(G)$. For each $X \in \mathcal{X}$, choose a character $\chi_{X}$ of $G$ such that $\chi_{X}=a_{X} \sigma_{X}$ for some nonzero constant $a_{X}$ (in particular, one could choose $\chi_{X}=\sigma_{X}$ ). We say that the partitions $\mathcal{K}$ and $\mathcal{X}$, along with the characters $\chi_{X}$, form a supercharacter theory of $G$ if

1. $|\mathcal{X}|=|\mathcal{K}|$,

2. the characters $\chi_{X}$ are constant on the members of $\mathcal{K}$, and

3. the set $\{1\} \in \mathcal{K}$.

The characters $\chi_{X}$ are referred to as supercharacters and the sets $K \in \mathcal{K}$ are called superclasses.

\subsection{Labeled set partitions}

A set partition $\nu$ of $[n]$ is a set of $\operatorname{arcs} i \frown j$ such that

1. $1 \leq i<j \leq n$, and

2. if $i \frown j, k \frown l \in \nu$, then $i=k$ if and only if $j=l$.

We will associate to a set partition a diagram consisting of nodes connected by arcs. For instance, if $\nu=\{1 \frown 2,2 \frown 6,5 \frown 7\}$ is a set partition of [7], then $\nu$ corresponds to the diagram

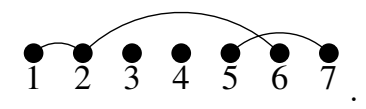

By considering connected components of the diagram, we can associate $\nu$ to a partition of the set $[n]$ (in this case $\{\{1,2,6\},\{3\},\{4\},\{5,7\}\})$, which is the standard definition of a set partition.

The advantage of our definition of a set partition is that we can attach labels to arcs. If $\mathbb{F}_{q}$ is the finite field with $q$ elements, an $\mathbb{F}_{q}$-set partition of $[n]$ is a set partition of $[n]$ with arcs labeled by nonzero elements of $\mathbb{F}_{q}$. We will associate to an $\mathbb{F}_{q}$-set partition a diagram consisting of nodes connected by labeled arcs. For instance, if $\nu=\{1 \stackrel{a}{\sim} 2,2 \stackrel{b}{\neg} 6,5 \stackrel{c}{\neg} 7\}$, then $\nu$ corresponds to the diagram

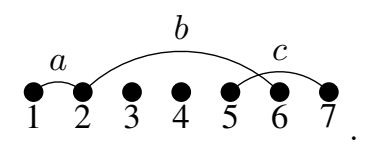

For a more complete discussion of labeled set partitions, see [Mar12]. 


\subsection{Algebra groups and pattern subgroups}

Let $\mathbb{F}$ be a field and let $\mathfrak{g}$ be a nilpotent associative algebra over $\mathbb{F}$. The algebra group $G$ associated to $\mathfrak{g}$ is the set of formal sums

$$
G=\{1+x \mid x \in \mathfrak{g}\}
$$

with multiplication defined by $(1+x)(1+y)=1+(x+y+x y)$ (see [Isa95]). As $\mathfrak{g}$ is nilpotent, elements in $G$ have inverses given by

$$
(1+x)^{-1}=1+\sum_{i=1}^{\infty}(-x)^{i} .
$$

We will often write $G=1+\mathfrak{g}$ to indicate that $G$ is the algebra group associated to $\mathfrak{g}$.

For example, if we define $U T_{n}\left(\mathbb{F}_{q}\right)$ to be the group of $n \times n$ upper triangular matrices over $\mathbb{F}_{q}$ with ones on the diagonal and $\mathfrak{u t}_{n}\left(\mathbb{F}_{q}\right)$ to be the algebra of $n \times n$ upper triangular matrices over $\mathbb{F}_{q}$ with zeroes on the diagonal, then $U T_{n}\left(\mathbb{F}_{q}\right)$ is the algebra group associated to $\mathfrak{u t}_{n}\left(\mathbb{F}_{q}\right)$. Let $\mathcal{P}$ be a poset on $[n]$ which is a sub-order of the usual total linear order. Corresponding to $\mathcal{P}$ are a pattern subgroup

$$
U_{\mathcal{P}}=\left\{g \in U T_{n}\left(\mathbb{F}_{q}\right) \mid g_{i j}=0 \text { unless } i \preceq_{\mathcal{P}} j\right\}
$$

and a pattern subalgebra

$$
\mathfrak{u}_{\mathcal{P}}=\left\{x \in \mathfrak{u t}_{n}\left(\mathbb{F}_{q}\right) \mid x_{i j}=0 \text { unless } i \preceq_{\mathcal{P}} j\right\} .
$$

Note that $U_{\mathcal{P}}$ is the algebra group corresponding to $\mathfrak{u}_{\mathcal{P}}$. For a more complete discussion of pattern subgroups, see [DT09].

In [DI08], Diaconis-Isaacs construct a supercharacter theory for an arbitrary finite algebra group $G=$ $1+\mathfrak{g}$. Note that $G$ acts on $\mathfrak{g}$ by left and right multiplication; there are corresponding actions of $G$ on the dual $\mathfrak{g}^{*}$ given by

$$
(g \lambda)(x)=\lambda\left(g^{-1} x\right) \quad \text { and }(\lambda g)(x)=\lambda\left(x g^{-1}\right),
$$

where $g \in G, \lambda \in \mathfrak{g}^{*}$, and $x \in \mathfrak{g}$. Let

$$
\begin{aligned}
f: G & \rightarrow \mathfrak{g} \\
g & \mapsto g-1
\end{aligned}
$$

and let $\theta: F_{q}^{+} \rightarrow \mathbb{C}^{\times}$be a nontrivial homomorphism. For $g \in G$ and $\lambda \in \mathfrak{g}^{*}$, define

$$
K_{g}=\{h \in G \mid f(h) \in G f(g) G\}
$$

and

$$
\chi_{\lambda}=\frac{|G \lambda|}{|G \lambda G|} \sum_{\mu \in G \lambda G} \theta \circ \mu \circ f .
$$

Theorem 2.1 (([DI08])) The partition of $G$ given by $\mathcal{K}=\left\{K_{g} \mid g \in G\right\}$, along with the set of characters $\left\{\chi_{\lambda} \mid \lambda \in \mathfrak{g}^{*}\right\}$, form a supercharacter theory of $G$. This supercharacter theory is independent of the choice of $\theta$.

The supercharacter theory is independent of $\theta$ in that the sets $\mathcal{K}$ and $\left\{\chi_{\lambda} \mid \lambda \in \mathfrak{u}^{*}\right\}$ do not depend on $\theta$. If a different $\theta$ is chosen, the $\chi_{\lambda}$ will be permuted. 


\section{Main result}

The main result of this paper is the construction of a supercharacter theory for certain subgroups of algebra groups which are defined by anti-involutions. In this section we define these groups and present the main result of the paper.

\subsection{Subgroups of algebra groups defined by anti-involutions}

For $q$ a power of a prime, let $\mathfrak{g}$ be a nilpotent associative algebra of finite dimension over $\mathbb{F}_{q}$. Let

$$
\begin{aligned}
\dagger: \mathfrak{g} & \rightarrow \mathfrak{g} \\
x & \mapsto x^{\dagger}
\end{aligned}
$$

be an involutive associative algebra antiautomorphism, and for $x \in \mathfrak{g}$ define $(1+x)^{\dagger}=1+x^{\dagger}$. Note that this makes $\dagger$ an involutive antiautomorphism of $G$. Define

$$
U=\left\{u \in G \mid u^{\dagger}=u^{-1}\right\}
$$

and

$$
\mathfrak{u}=\left\{x \in \mathfrak{g} \mid x^{\dagger}=-x\right\}
$$

Note that $\mathfrak{u}$ is not an associative algebra, although it is closed under the Lie bracket. The motivating examples of groups defined in this manner are the unipotent orthogonal, symplectic, and unitary groups in odd characteristic.

\subsection{Springer morphisms}

In order to utilize the Lie algebra structure of $\mathfrak{u}$ in studying $U$, we would like a bijection between $U$ and $\mathfrak{u}$ that preserves useful properties. In the case of an algebra group $G$, we can use the map $g \mapsto g-1$ to relate $G$ to $\mathfrak{g}$. In general, however, it is not the case that $U=1+\mathfrak{u}$, so we need a variation on this map. André-Neto define a bijection from $U$ to $\mathfrak{u}$ in [AN06], however we require a map that is invariant under the adjoint action of $U$.

Given an algebra group $G=1+\mathfrak{g}$ and a map $\nmid$ as above, we define a Springer morphism $f: G \rightarrow \mathfrak{g}$ to be a bijection such that

1. $f(U)=\mathfrak{u}$ and $f^{-1}(\mathfrak{u})=U$.

2. There exist $a_{i} \in \mathbb{F}_{q}$ such that $f(1+x)=x+\sum_{i=2}^{\infty} a_{i} x^{i}$.

Springer morphisms are introduced by Springer and Steinberg in [SS70] (III, 3.12) and are utilized by Kawanaka in [Kaw85]. The following lemma is easy to verify directly.

Lemma 3.1 Let $q$ be a power of an odd prime $p$, let $G=1+\mathfrak{g}$ be any algebra group, and let $\dagger$ be any anti-involution of $\mathfrak{g}$. Then the map

$$
f(1+x)=2 x(x+2)^{-1} .
$$

is a Springer morphism.

The map $f$ is a constant multiple of the map $1+x \mapsto x(x+2)^{-1}$, which is often referred to as the Cayley map (see, for instance, [Kaw85]). This lemma allows us to assume the existence of a Springer morphism if we are working in odd characteristic, which we will do for the remainder of the paper. 


\subsection{Main theorem}

Let $q$ be a power of an odd prime, and let $G=1+\mathfrak{g}$ be a pattern subgroup of $U T_{n}\left(\mathbb{F}_{q^{k}}\right)$ for some $n$ and $k$. For $1 \leq i \leq n$, define $\bar{i}=n+1-i$. We consider $\mathfrak{g}$ as an $\mathbb{F}_{q}$-algebra; let $\dagger$ be an anti-involution of $\mathfrak{g}$ such that $\left(\alpha e_{i j}\right)^{\dagger} \in \mathbb{F}_{q^{k}}^{\times} e_{\bar{j} \bar{i}}$ for all $\alpha \in \mathbb{F}_{q^{k}}^{\times}$. In other words, $\dagger$ reflects the entries of elements of $\mathfrak{g}$ across the antidiagonal, up to a constant multiple. The antiautomorphisms which define the orthogonal, symplectic and unitary groups all have this property. Let

$$
U=\left\{u \in G \mid u^{\dagger}=u^{-1}\right\}
$$

and

$$
\mathfrak{u}=\left\{x \in \mathfrak{g} \mid x^{\dagger}=-x\right\}
$$

as above. Let $f$ be any Springer morphism and let $\theta: \mathbb{F}_{q}^{+} \rightarrow \mathbb{C}^{\times}$be a nontrivial homomorphism. For $g \in G, x \in \mathfrak{u}$ and $\lambda \in \mathfrak{u}^{*}$, let $g \cdot x=g x g^{\dagger}$ and $(g \cdot \lambda)(x)=\lambda\left(g^{-1} \cdot x\right)$. For $\lambda \in \mathfrak{u}^{*}$ and $u \in U$, define

$$
K_{u}=\{v \in U \mid f(v) \in G \cdot f(u)\}
$$

and

$$
\chi_{\lambda}=\frac{1}{n_{\lambda}} \sum_{\mu \in G \cdot \lambda} \theta \circ \mu \circ f
$$

where $n_{\lambda}$ is a constant determined by $\lambda$ (and independent of the choice of $\lambda$ as orbit representative). As in [DI08], $n_{\lambda}$ can be written in terms of the sizes of orbits of group actions. If we let $H$ be the subgroup of $G$ defined by

$$
H=\left\{h \in G \mid h_{i j}=0 \text { if } j \leq \frac{n}{2}\right\}
$$

then

$$
n_{\lambda}=\frac{|G \cdot \lambda|}{|H \cdot \lambda|} .
$$

Theorem 3.2 The partition of $U$ given by $\mathcal{K}=\left\{K_{u} \mid u \in U\right\}$, along with the set of characters $\left\{\chi_{\lambda} \mid\right.$ $\left.\lambda \in \mathfrak{u}^{*}\right\}$, form a supercharacter theory of $U$. This supercharacter theory is independent of the choice of $\theta$ and $f$.

The supercharacter theory is independent of $\theta$ and $f$ in that the sets $\mathcal{K}$ and $\left\{\chi_{\lambda} \mid \lambda \in \mathfrak{u}^{*}\right\}$ do not depend on these functions. If a different $\theta$ is chosen or condition (2) in the definition of a Springer morphism is relaxed to allow for other $x$ coefficients, the $\chi_{\lambda}$ will be permuted. The supercharacter theory is also independent of the choice of subfield of $\mathbb{F}_{q^{k}}$; that is, if $\mathbb{F}$ is any subfield of $\mathbb{F}_{q^{k}}$ and $\dagger$ is an antiautomorphism of $\mathfrak{g}$ when viewed as an $\mathbb{F}$-algebra, we get the same supercharacter theory as by considering $\mathfrak{g}$ as an $\mathbb{F}_{q}$-algebra. The following result allows us to relate our supercharacter theories to those of André and Neto.

Theorem 3.3 The superclasses of $U$ are exactly the sets of the form $U \cap K_{g}$, where $K_{g}$ is some superclass of $G$. 


\section{Supercharacter theories of unipotent orthogonal and symplec- tic groups}

In this section we use Theorem 3.2 to construct supercharacter theories for two families of groups.

\subsection{Supercharacter theories of unipotent orthogonal groups}

Let $J$ be the $n \times n$ matrix with ones on the antidiagonal and zeroes elsewhere, and let $x^{t}$ denote the transpose of a matrix $x$. For $q$ a power of an odd prime, define

$$
O_{n}\left(\mathbb{F}_{q}\right)=\left\{g \in G L_{n}\left(\mathbb{F}_{q}\right) \mid g^{-1}=J g^{t} J\right\}
$$

along with the corresponding Lie algebra

$$
\mathfrak{o}_{n}\left(\mathbb{F}_{q}\right)=\left\{x \in \mathfrak{g l}_{n}\left(\mathbb{F}_{q}\right) \mid-x=J x^{t} J\right\} .
$$

Let $U T_{n}\left(\mathbb{F}_{q}\right)$ be the set of unipotent upper triangular matrices of $G L_{n}\left(\mathbb{F}_{q}\right)$ and $\mathfrak{u t}_{n}\left(\mathbb{F}_{q}\right)$ be the set of strictly upper triangular matrices of $\mathfrak{g l}_{n}\left(\mathbb{F}_{q}\right)$. Define

$$
\begin{aligned}
U O_{n}\left(\mathbb{F}_{q}\right) & =U T_{n}\left(\mathbb{F}_{q}\right) \cap O_{n}\left(\mathbb{F}_{q}\right) \text { and } \\
\mathfrak{u o}_{n}\left(\mathbb{F}_{q}\right) & =\mathfrak{u t}_{n}\left(\mathbb{F}_{q}\right) \cap \mathfrak{o}_{n}\left(\mathbb{F}_{q}\right) .
\end{aligned}
$$

Define an antiautomorphism $\dagger$ of $\mathfrak{u t}_{n}\left(\mathbb{F}_{q}\right)$ by $x^{\dagger}=J x^{t} J$. Note that $\dagger$ satisfies the conditions required by Theorem 3.2. and furthermore

$$
\begin{aligned}
U O_{n}\left(\mathbb{F}_{q}\right) & =\left\{g \in U T_{2 n}\left(\mathbb{F}_{q}\right) \mid g^{-1}=g^{\dagger}\right\} \text { and } \\
\mathfrak{u o}_{n}\left(\mathbb{F}_{q}\right) & =\left\{x \in \mathfrak{u t}_{2 n}\left(\mathbb{F}_{q}\right) \mid-x=x^{\dagger}\right\} .
\end{aligned}
$$

Define $K_{u}$ and $\chi_{\lambda}$ as in 1 and 2 with $U=U O_{n}\left(\mathbb{F}_{q}\right)$ and $\mathfrak{u}=\mathfrak{u o}_{n}\left(\mathbb{F}_{q}\right)$. By Theorem 3.2, there is a supercharacter theory of $U O_{n}\left(\mathbb{F}_{q}\right)$ with superclasses $\left\{K_{u}\right\}$ and supercharacters $\left\{\chi_{\lambda}\right\}$.

In [AN09a], André-Neto construct a supercharacter theory of $U O_{n}\left(\mathbb{F}_{q}\right)$. They show that their superclasses are the sets of the form $U O_{n}\left(\mathbb{F}_{q}\right) \cap K_{g}$, where $K_{g}$ is a superclass of $U T_{n}\left(\mathbb{F}_{q}\right)$ under the algebra group supercharacter theory. In particular, the following theorem follows from Theorem 3.3 .

Theorem 4.1 The supercharacter theory of $U O_{n}\left(\mathbb{F}_{q}\right)$ defined above coincides with that of André-Neto in [AN09a].

We can also construct supercharacter theories of certain subgroups of $U O_{n}\left(\mathbb{F}_{q}\right)$ using this method. We will call a poset $\mathcal{P}$ symmetric if $i \preceq_{\mathcal{P}} j$ implies that $\bar{j} \preceq_{\mathcal{P}} \bar{i}$ (recall that $\bar{i}=n-i+1$ ). The antiautomorphism $\dagger$ as defined above restricts to an antiautomorphism of $U_{\mathcal{P}}$ for any symmetric poset. Furthermore,

$$
\begin{aligned}
U O_{n}\left(\mathbb{F}_{q}\right) \cap U_{\mathcal{P}} & =\left\{g \in U_{\mathcal{P}} \mid g^{-1}=g^{\dagger}\right\} \text { and } \\
\mathfrak{u o}_{n}\left(\mathbb{F}_{q}\right) \cap \mathfrak{u}_{\mathcal{P}} & =\left\{x \in \mathfrak{u}_{\mathcal{P}} \mid-x=x^{\dagger}\right\} .
\end{aligned}
$$

Define $K_{u}$ and $\chi_{\lambda}$ as in 1 and 2 with $U=U O_{n}\left(\mathbb{F}_{q}\right) \cap U_{\mathcal{P}}$ and $\mathfrak{u}=\mathfrak{u o}_{n}\left(\mathbb{F}_{q}\right) \cap \mathfrak{u}_{\mathcal{P}}$. By Theorem 3.2 . there is a supercharacter theory of $U O_{n}\left(\mathbb{F}_{q}\right) \cap U_{\mathcal{P}}$ with superclasses $\left\{K_{u}\right\}$ and supercharacters $\left\{\chi_{\lambda}\right\}$. By Theorem 3.3 the superclasses are of the form $K_{g} \cap U O_{n}\left(\mathbb{F}_{q}\right)$ where $K_{g}$ is a superclass of $U_{\mathcal{P}}$ in the algebra group supercharacter theory. In particular, if $U$ is the unipotent radical of a parabolic subgroup of $O_{n}\left(\mathbb{F}_{q}\right)$ then $U=U O_{n}\left(\mathbb{F}_{q}\right) \cap U_{\mathcal{P}}$ for some symmetric poset $\mathcal{P}$. 
There are two important examples of a subgroup obtained from a symmetric poset in type $D$. First, let $P$ be the symmetric poset on $[2 n]$ defined by

$$
i \preceq_{\mathcal{P}} j \text { if } i \leq j \text { and }(i, j) \neq(n, n+1) .
$$

Then $U O_{2 n}\left(\mathbb{F}_{q}\right) \cap U_{\mathcal{P}}=U O_{2 n}\left(\mathbb{F}_{q}\right)$, and we get a second supercharacter theory of $U O_{2 n}\left(\mathbb{F}_{q}\right)$ which is at least as fine as the one originally defined. This new supercharacter theory is in fact strictly finer than the original; the elements $e_{1, n}-e_{n+1,2 n}$ and $\left(e_{1, n}-e_{n+1,2 n}\right)+\left(e_{1, n+1}-e_{n, 2 n}\right)$ of $\mathfrak{u}$ are in the same orbit under the action of $U T_{2 n}\left(\mathbb{F}_{q}\right)$ on $\mathfrak{u o}_{2 n}\left(\mathbb{F}_{q}\right)$, but in different orbits under the action of $U_{\mathcal{P}}$ on $\mathfrak{u o}_{2 n}\left(\mathbb{F}_{q}\right)$.

We can also consider the poset $\mathcal{P}$ on $[2 n]$ defined by

$$
i \preceq \mathcal{P} j \text { if } i \leq j \leq n \text { or } n+1 \leq i \leq j .
$$

In this case, $U O_{2 n}\left(\mathbb{F}_{q}\right) \cap U_{\mathcal{P}} \cong U T_{n}\left(\mathbb{F}_{q}\right)$, and the supercharacter theory obtained is the algebra group supercharacter theory.

\subsection{Supercharacter theories of unipotent symplectic groups}

Let $J$ be the $n \times n$ matrix with ones on the antidiagonal and zeroes elsewhere, and define

$$
\Omega=\left(\begin{array}{cc}
0 & -J \\
J & 0
\end{array}\right)
$$

For $q$ a power of an odd prime, define

$$
S p_{2 n}\left(\mathbb{F}_{q}\right)=\left\{g \in G L_{2 n}\left(\mathbb{F}_{q}\right) \mid g^{-1}=-\Omega g^{t} \Omega\right\}
$$

along with the corresponding Lie algebra

$$
\mathfrak{s p}_{2 n}\left(\mathbb{F}_{q}\right)=\left\{x \in \mathfrak{g l}_{2 n}\left(\mathbb{F}_{q}\right) \mid-x=-\Omega x^{t} \Omega\right\} .
$$

Let $U T_{2 n}\left(\mathbb{F}_{q}\right)$ be the set of unipotent upper triangular matrices of $G L_{2 n}\left(\mathbb{F}_{q}\right)$, and $\mathfrak{u t}_{2 n}\left(\mathbb{F}_{q}\right)$ be the set of strictly upper triangular matrices of $\mathfrak{g l}_{2 n}\left(\mathbb{F}_{q}\right)$. Define

$$
\begin{aligned}
U S p_{2 n}\left(\mathbb{F}_{q}\right) & =U T_{2 n}\left(\mathbb{F}_{q}\right) \cap S p_{2 n}\left(\mathbb{F}_{q}\right) \text { and } \\
\mathfrak{u s p}_{2 n}\left(\mathbb{F}_{q}\right) & =\mathfrak{u t}_{2 n}\left(\mathbb{F}_{q}\right) \cap \mathfrak{s p}_{2 n}\left(\mathbb{F}_{q}\right) .
\end{aligned}
$$

Define an antiautomorphism $\dagger$ of $\mathfrak{u t}_{2 n}\left(\mathbb{F}_{q}\right)$ by $x^{\dagger}=-\Omega x^{t} \Omega$. Note that $\dagger$ satisfies the conditions required by Theorem 3.2 , and furthermore

$$
\begin{aligned}
U S p_{2 n}\left(\mathbb{F}_{q}\right) & =\left\{g \in U T_{2 n}\left(\mathbb{F}_{q}\right) \mid g^{-1}=g^{\dagger}\right\} \text { and } \\
\mathfrak{u s p}_{2 n}\left(\mathbb{F}_{q}\right) & =\left\{x \in \mathfrak{u t}_{2 n}\left(\mathbb{F}_{q}\right) \mid-x=x^{\dagger}\right\} .
\end{aligned}
$$

Define $K_{u}$ and $\chi_{\lambda}$ as in 1 and 2 with $U=U S p_{2 n}\left(\mathbb{F}_{q}\right)$ and $\mathfrak{u}=\mathfrak{u s p}_{2 n}\left(\mathbb{F}_{q}\right)$. By Theorem 3.2 there is a supercharacter theory of $U S p_{2 n}\left(\mathbb{F}_{q}\right)$ with superclasses $\left\{K_{u}\right\}$ and supercharacters $\left\{\chi_{\lambda}\right\}$. 
In [AN09a], André-Neto have also constructed supercharacter theories of $U S p_{2 n}\left(\mathbb{F}_{q}\right)$. As was the case with the unipotent orthogonal groups, their supercharacter theories are the sets of the form $U \operatorname{Sp}_{2 n}\left(\mathbb{F}_{q}\right) \cap$ $K_{g}$, where $K_{g}$ is a superclass of $U T_{2 n}\left(\mathbb{F}_{q}\right)$ under the algebra group supercharacter theory. In particular, the following theorem follows from Theorem 3.3 .

Theorem 4.2 The supercharacter theory of $U S p_{2 n}\left(\mathbb{F}_{q}\right)$ defined above coincides with that of André-Neto in [AN09a].

We mention that we can also construct supercharacter theories of certain subgroups of $U S p_{2 n}\left(\mathbb{F}_{q}\right)$ just as we did for $U O_{n}\left(\mathbb{F}_{q}\right)$. We skip this construction as it is identical to the orthogonal case.

\section{Supercharacter theories of the unipotent unitary groups}

Let $q$ be a power of an odd prime, and for $x \in \mathfrak{g l}_{n}\left(\mathbb{F}_{q^{2}}\right)$, define $\bar{x}$ by $(\bar{x})_{i j}=\left(x_{i j}\right)^{q}$. Let

$$
\begin{aligned}
U_{n}\left(\mathbb{F}_{q^{2}}\right) & =\left\{g \in G L_{n}\left(\mathbb{F}_{q^{2}}\right) \mid g^{-1}=J \bar{g}^{t} J\right\} \text { and } \\
\mathfrak{u}_{n}\left(\mathbb{F}_{q^{2}}\right) & =\left\{x \in \mathfrak{g l}_{n}\left(\mathbb{F}_{q^{2}}\right) \mid-x=J \bar{x}^{t} J\right\},
\end{aligned}
$$

and let

$$
\begin{aligned}
U U_{n}\left(\mathbb{F}_{q^{2}}\right) & =U_{n}\left(\mathbb{F}_{q^{2}}\right) \cap U T_{n}\left(\mathbb{F}_{q^{2}}\right) \text { and } \\
\mathfrak{u u}_{n}\left(\mathbb{F}_{q^{2}}\right) & =\mathfrak{u}_{n}\left(\mathbb{F}_{q^{2}}\right) \cap \mathfrak{u t}_{n}\left(\mathbb{F}_{q^{2}}\right) .
\end{aligned}
$$

The group $U_{n}\left(\mathbb{F}_{q^{2}}\right)$ is the group of unitary $n \times n$ matrices over $\mathbb{F}_{q^{2}}$. In this section we construct a supercharacter theory of $U U_{n}\left(\mathbb{F}_{q^{2}}\right)$ using Theorem 3.2 and calculate the values of the supercharacters on the superclasses.

\subsection{Construction}

The map $x^{\dagger}=J \bar{x}^{t} J$ defines an antiautomorphism of $\mathfrak{u t}_{n}\left(\mathbb{F}_{q^{2}}\right)$ if we consider $\mathfrak{u t}_{n}\left(\mathbb{F}_{q^{2}}\right)$ as an $\mathbb{F}_{q^{-}}$-algebra. This involution satisfies the conditions required by Theorem 3.2 and furthermore

$$
\begin{aligned}
U U_{n}\left(\mathbb{F}_{q^{2}}\right) & =\left\{g \in U T_{n}\left(\mathbb{F}_{q^{2}}\right) \mid g^{-1}=g^{\dagger}\right\} \text { and } \\
\mathfrak{u u}_{n}\left(\mathbb{F}_{q^{2}}\right) & =\left\{x \in \mathfrak{u t}_{n}\left(\mathbb{F}_{q^{2}}\right) \mid-x=x^{\dagger}\right\} .
\end{aligned}
$$

Define $K_{u}$ and $\chi_{\lambda}$ as in 1 and 2 with $U=U U_{n}\left(\mathbb{F}_{q^{2}}\right)$ and $\mathfrak{u}=\mathfrak{u u}_{n}\left(\mathbb{F}_{q^{2}}\right)$. By Theorem 3.2 there is a supercharacter theory of $U U_{n}\left(\mathbb{F}_{q^{2}}\right)$ with superclasses $\left\{K_{u}\right\}$ and supercharacters $\left\{\chi_{\lambda}\right\}$.

As with the orthogonal and symplectic cases, by Theorem 3.3 the superclasses are of the form $K_{g} \cap$ $U U_{n}\left(\mathbb{F}_{q^{2}}\right)$ where $K_{g}$ is a superclass of $U T_{n}\left(\mathbb{F}_{q^{2}}\right)$ under the algebra group supercharacter theory.

We mention that we can also construct supercharacter theories of certain subgroups of $U U_{n}\left(\mathbb{F}_{q}\right)$ just as we did for $U O_{n}\left(\mathbb{F}_{q}\right)$. We skip this construction as it is identical to the orthogonal case. 


\subsection{Superclasses and supercharacters}

In this section we describe the superclasses and supercharacters of $U=U U_{n}\left(\mathbb{F}_{q^{2}}\right)$ in terms of labeled set partitions. Recall that, for $1 \leq i \leq n$, we define $\bar{i}=n+1-i$. A twisted $\mathbb{F}_{q}$-set partition will refer to

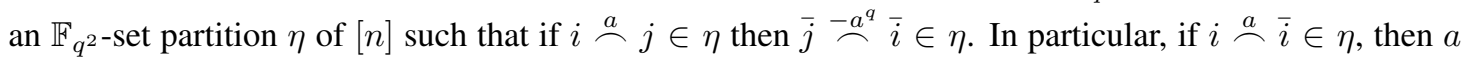
satisfies $a^{q}+a=0$. For more on labeled set partitions, see [Mar12].

Lemma 5.1 Each superclass of $U$ contains exactly one element $u$ with the property that $f(u)$ has at most one nonzero entry in each row and column.

To each twisted $\mathbb{F}_{q}$-set partition $\eta$ we assign the element $x_{\eta} \in \mathfrak{u}$ defined by

$$
\left(x_{\eta}\right)_{i j}= \begin{cases}a & \text { if } i \stackrel{a}{\sim} j \in \eta \\ 0 & \text { else. }\end{cases}
$$

and the element $u_{\eta} \in U$ such that $f\left(u_{\eta}\right)=x_{\eta}$. Note that $x_{\eta}$ is in fact an element of $\mathfrak{u}$ and has at most one entry in each nonzero row and column.

Corollary 5.2 The elements

$$
\left\{u_{\eta} \mid \eta \text { is a twisted } \mathbb{F}_{q} \text {-partition }\right\}
$$

are a set of superclass representatives.

As there are equal numbers of superclasses and supercharacters, the supercharacters can also be parameterized by twisted $\mathbb{F}_{q}$-set partitions. Given a twisted $\mathbb{F}_{q}$-set partition, define $\lambda_{\eta} \in \mathfrak{u}^{*}$ by

$$
\lambda_{\eta}(x)=\sum_{i \stackrel{a}{\sim} j \in \eta} a x_{i j} .
$$

Lemma 5.3 The set

$$
\left\{\lambda_{\eta} \mid \eta \text { is a twisted } \mathbb{F}_{q} \text {-partition }\right\}
$$

is a set of orbit representatives for the action of $U T_{n}\left(\mathbb{F}_{q^{2}}\right)$ on $\mathfrak{u}^{*}$.

For a twisted $\mathbb{F}_{q^{-}}$-set partition, we define $\chi^{\eta}=\chi_{\lambda_{\eta}}$.

Corollary 5.4 The superclasses and supercharacters are given by

$$
\left\{K_{u_{\eta}} \mid \eta \text { is a twisted } \mathbb{F}_{q} \text {-partition }\right\}
$$

and

$$
\left\{\chi^{\eta} \mid \eta \text { is a twisted } \mathbb{F}_{q} \text {-partition }\right\} .
$$

\subsection{Supercharacter values on superclasses}

The goal of this section is to calculate $\chi^{\eta}\left(u_{\nu}\right)$, where $\eta$ and $\nu$ are twisted $\mathbb{F}_{q}$-set partitions. We will call a supercharacter elementary if it corresponds to a twisted $\mathbb{F}_{q}$-set partition of the form $\eta=\left\{i \stackrel{a}{\sim} j \cup \bar{j} \stackrel{-a^{q}}{\sim} \bar{i}\right\}$ with $i \neq \bar{j}$ or of the form $\eta=\{i \stackrel{a}{\sim} \bar{i}\}$ with $a^{q}+a=0$. In order to simplify calculations, we will show that every supercharacter can be written as a product of distinct elementary supercharacters. This is analogous to the method used in types $A, B, C$ and $D$ (see [And02, AN09a]). 
For a twisted $\mathbb{F}_{q}$-set partition $\eta$, we can write $\eta$ as a disjoint union of twisted $\mathbb{F}_{q}$-set partitions of the form $\left\{i \stackrel{a}{\sim} j \cup \bar{j} \stackrel{-a^{q}}{\sim} \bar{i}\right\}$ with $i \neq \bar{j}$ or of the form $\{i \stackrel{a}{\sim} \bar{i}\}$ with $a^{q}+a=0$. In other words, there exists $m$ such that

$$
\eta=\bigsqcup_{r=1}^{m} \eta_{r}
$$

with each $\eta_{r}$ of the described form.

For two characters $\chi$ and $\psi$, define their product by $(\chi \psi)(u)=\chi(u) \psi(u)$.

Lemma 5.5 With notation as above,

$$
\chi^{\eta}=\prod_{r=1}^{m} \chi^{\eta_{r}} .
$$

We now calculate the values of the supercharacters on the superclasses. First, we determine the dimensions of the elementary supercharacters.

Lemma 5.6 Let $\eta=\left\{i \stackrel{a}{\sim} j \cup \bar{j} \stackrel{-a^{q}}{\sim} \bar{i}\right\}$ (with $i \neq \bar{j}$ ) be a twisted $\mathbb{F}_{q}$-set partition; then

$$
\chi^{\eta}(1)= \begin{cases}q^{2(j-i-1)} & \text { if } n \text { is even } \\ q^{2(j-i-1)} & \text { if } n \text { is odd and } j \leq \frac{n+1}{2} \\ q^{2(j-i)} & \text { if } n \text { is odd and } j>\frac{n+1}{2} .\end{cases}
$$

Let $\eta=\left\{i \stackrel{a}{\sim} \bar{i}\right.$ (with $\left.i \leq \frac{n+1}{2}\right)$ be a twisted $\mathbb{F}_{q}$-set partition; then

$$
\chi^{\eta}(1)= \begin{cases}q^{2(n-2 i)} & \text { if } n \text { is even } \\ q^{2(n+1-2 i)} & \text { if } n \text { is odd }\end{cases}
$$

We mention that the dimension of an arbitrary supercharacter can be calculated by applying Lemma 5.5 Next we calculate the value of a supercharacter on a superclass.

Theorem 5.7 Let $\eta$ and $\nu$ be twisted $\mathbb{F}_{q}$-set partitions. Then

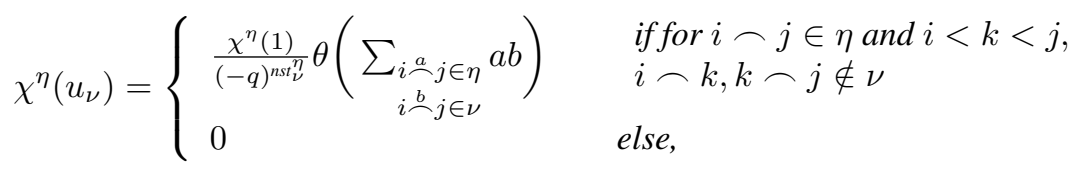

where $n s t_{\nu}^{\eta}=|\{i<j<k<l \mid j \frown k \in \nu, i \frown l \in \eta\}|$.

By Lemma 5.5 , the proof reduces to proving that the theorem holds in the case that $\chi_{\eta}$ is an elementary supercharacter. Note that in the above formula the sum of the terms $a b$ is an element of $\mathbb{F}_{q}$, even though each individual term might not be. This formula is identical to the type $A$ supercharacter formula (which was first derived by André, and can be found in [Thi10]), except with $-q$ replacing $q$ everywhere (note that the supercharacter degrees are all powers of $q^{2}$ ). This idea that information about the unitary group can be obtained from information about the general linear group by replacing $q$ with $-q$ is referred to as Ennola duality (see [Kaw85]). 


\section{References}

[AN06] C. A. M. André and A. M. Neto. Super-characters of finite unipotent groups of types $B_{n}, C_{n}$ and $D_{n}$. J. Algebra, 305(1):394-429, 2006.

[AN09a] C. A. M. André and A. M. Neto. A supercharacter theory for the Sylow $p$-subgroups of the finite symplectic and orthogonal groups. J. Algebra, 322(4):1273-1294, 2009.

[AN09b] C. A. M. André and A. M. Neto. Supercharacters of the Sylow $p$-subgroups of the finite symplectic and orthogonal groups. Pacific J. Math., 239(2):201-230, 2009.

[And95] C. A. M. André. Basic characters of the unitriangular group. J. Algebra, 175(1):287-319, 1995.

[And02] C. A. M. André. Basic characters of the unitriangular group (for arbitrary primes). Proc. Amer. Math. Soc., 130(7):1943-1954 (electronic), 2002.

[DI08] P. Diaconis and I. M. Isaacs. Supercharacters and superclasses for algebra groups. Trans. Amer. Math. Soc., 360(5):2359-2392, 2008.

[DT09] P. Diaconis and N. Thiem. Supercharacter formulas for pattern groups. Trans. Amer. Math. Soc., 361(7):3501-3533, 2009.

[GKP +90$]$ P. M. Gudivok, Yu. V. Kapitonova, S. S. Polyak, V. P. Rud'ko, and A. I. Tsitkin. Classes of conjugate elements of a unitriangular group. Kibernetika (Kiev), (1):40-48, 133, 1990.

[Isa95] I. M. Isaacs. Characters of groups associated with finite algebras. J. Algebra, 177(3):708-730, 1995.

[Kaw85] N. Kawanaka. Generalized Gel'fand-Graev representations and Ennola duality. In Algebraic groups and related topics (Kyoto/Nagoya, 1983), volume 6 of Adv. Stud. Pure Math., pages 175-206. North-Holland, Amsterdam, 1985.

[Mar12] E. Marberg. Actions and identities on set partitions. Electron. J. Combin., 19(1):Paper 28, 31, 2012.

[SS70] T. A. Springer and R. Steinberg. Conjugacy classes. In Seminar on Algebraic Groups and Related Finite Groups (The Institute for Advanced Study, Princeton, N.J., 1968/69), Lecture Notes in Mathematics, Vol. 131, pages 167-266. Springer, Berlin, 1970.

[Thi10] N. Thiem. Branching rules in the ring of superclass functions of unipotent upper-triangular matrices. J. Algebraic Combin., 31(2):267-298, 2010. 